\title{
Time-periodic solutions for a driven sixth-order Cahn-Hilliard type equation
}

\section{Changchun Liu*, Aibo Liu and Hui Tang}

\author{
"Correspondence: liucc@jlu.edu.cn \\ Department of Mathematics, Jilin \\ University, Changchun, 130012 \\ China
}

\begin{abstract}
We study a driven sixth-order Cahn-Hilliard type equation which arises naturally as a continuum model for the formation of quantum dots and their faceting. Based on the Leray-Schauder fixed point theorem, we prove the existence of time-periodic solutions.

MSC: 35B10; 35K55; 35K65

Keywords: sixth-order Cahn-Hilliard equation; time-periodic solution; existence; Campanato space
\end{abstract}

\section{Introduction}

In this paper, we are concerned with the following problem for the sixth-order CahnHilliard type equation:

$$
\begin{aligned}
& \frac{\partial u}{\partial t}-\gamma D^{6} u=D^{4} \psi(u, t)+v u D u+f(x, t), \quad(x, t) \in Q, \\
& \left.u\right|_{x=0,1}=\left.D^{2} u\right|_{x=0,1}=\left.D^{4} u\right|_{x=0,1}=0, \quad t \geq 0, \\
& u(x, 0)=u(x, T), \quad x \in(0,1),
\end{aligned}
$$

where $Q \equiv(0,1) \times(0,+\infty), D=\frac{\partial}{\partial x}, \psi(u, t)=-a(t) u^{3}+b(t) u, a(t)$ and $b(t)$ are Hölder continuous functions defined on $\mathbb{R}^{+}$with period $T, f(x, t)$ belongs to the space $C^{\alpha, \frac{\alpha}{4}}(\bar{Q})$ for some $\alpha \in(0,1)$ with $f(x, 0)=f(x, T)$. Furthermore, we assume that $\underline{M} \leq a(t) \leq \bar{M}$, $|b(t)| \leq N,\left|a^{\prime}(t)\right| \leq L,\left|b^{\prime}(t)\right| \leq \Lambda$, where $\gamma, v, \underline{M}, \bar{M}, N, L$ and $\Lambda$ are positive constants.

Equation (1.1) with $f(x, t)=0$ arises naturally as a continuum model for the formation of quantum dots and their faceting; see [1]. It can also be used to describe competition and exclusion of biological population [2]. If we consider that the perturbation function $f(x, t)$ (for example, source) has the influence, then we obtain equation (1.1).

Korzec et al. [3] studied equation (1.1) with $f(x, t)=0$. New types of stationary solutions of one-dimensional driven sixth-order Cahn-Hilliard type equation (1.1) are derived by an extension of the method of matched asymptotic expansions that retains exponentially small terms. Liu et al. [4] proved that equation (1.1) with $f(x, t)=0$ possesses a global attractor in the $H^{k}(k \geq 0)$ space, which attracts any bounded subset of $H^{k}(\Omega)$ in the $H^{k}$-norm.

During the past years, many authors have paid much attention to other sixth-order thin film equations such as the existence, uniqueness and regularity of the solutions [5-7]. 
However, as far as we know, there are few investigations concerned with the time-periodic solutions of equation (1.1), even though there is some literature for population models and Cahn-Hilliard [8, 9]. In fact, it is natural to consider the time-periodic solutions of equation (1.1) when it is used to describe the models of the growth and dispersal in the population which is sensitive to time-periodic factors (for example, seasons). In this paper, we prove the existence of time-periodic solutions of problem (1.1)-(1.3) based on the framework of the Leray-Schauder fixed point theorem which can be found in any standard textbook of PDE (see, for example, [10]). For this purpose, we first introduce an operator $\mathcal{L}$ by considering a linear sixth-order equation with a parameter $\sigma \in[0,1]$. After verifying the compactness of the operator and some necessary a priori estimates for the solutions, we then obtain a fixed point of the operator in a suitable functional space with $\sigma=1$, which is the desired solution of problem (1.1)-(1.3).

The main difficulties for treating problem (1.1)-(1.3) are caused by the nonlinearity of both the fourth-order term and the convective factors. The main method that we use is based on the Schauder-type a priori estimates, which here are obtained by means of a modified Campanato space. We note that the Campanato spaces have been widely used for the discussion of partial regularity of solutions of parabolic systems of second order and fourth order. So, in the following section we give a detailed description and the associated properties of such a space, and subsequently, in the next section we prove the existence of classical time-periodic solutions of problem (1.1)-(1.3).

\section{Hölder norm estimates}

Let $Q_{T}=(0,1) \times(0, T), y_{0}=\left(x_{0}, t_{0}\right) \in \bar{Q}_{T}$. For any fixed $R>0$, we define

$$
\begin{aligned}
& B_{R}=B_{R}\left(x_{0}\right)=\left(x_{0}-R, x_{0}+R\right), \quad I_{R}=I_{R}\left(t_{0}\right)=\left(t_{0}-R^{6}, t_{0}+R^{6}\right), \\
& Q_{R}=Q_{R}\left(y_{0}\right)=I_{R}\left(t_{0}\right) \times B_{R}\left(x_{0}\right), \quad S_{R}=Q_{R} \cap Q_{T}, \\
& E_{R}=E_{R}\left(x_{0}\right)=B_{R}\left(x_{0}\right) \cap(0,1), \quad J_{R}=J_{R}\left(t_{0}\right)=I_{R}\left(t_{0}\right) \cap(0, T) .
\end{aligned}
$$

Let $u$ be a function defined on $Q_{T}$, and set

$$
u_{R}=u_{y_{0}, R}=\frac{1}{\left|S_{R}\right|} \iint_{S_{R}} u d x d t, \quad \hat{u}_{R}=\hat{u}_{y_{0}, R}= \begin{cases}u_{R} & \text { if } Q_{R} \cap \partial_{p} Q_{T}=\emptyset, \\ 0 & \text { if } Q_{R} \cap \partial_{p} Q_{T} \neq \emptyset,\end{cases}
$$

where $\partial_{p} Q_{T}$ denotes the parabolic boundary of $Q_{T}$ and $\left|S_{R}\right|$ denotes the area of $S_{R}$.

For any $u \in C\left(\bar{Q}_{T}\right)$ and $\lambda>0$, define

$$
M^{2}[u]=\sup _{y_{0} \in \bar{Q}_{T}} \sup _{0<R \leq R_{0}} \frac{1}{R^{\lambda}} \iint_{S_{R}\left(y_{0}\right)}\left|u(x, t)-\hat{u}_{y_{0}, R}\right|^{2} d x d t,
$$

where $R_{0}=\operatorname{diam} Q_{T}$. By the space $\mathcal{L}^{2, \lambda}\left(Q_{T}\right)$ we mean the subset of $C\left(\bar{Q}_{T}\right)$, each element of which satisfies $M[u]<+\infty$. For $u \in \mathcal{L}_{0}^{2, \lambda}$, its norm is defined as

$$
\|u\|_{\mathcal{L}_{0}^{2, \lambda}}\left(Q_{T}\right)=\sup _{Q_{T}}|u(x, t)|+M[u] .
$$

Now, we give some useful lemmas. 
Lemma 2.1 [11] Let $\lambda>7$,

$$
\|u\|_{C^{\alpha, \frac{\alpha}{6}}} \leq C(\lambda)\|u\|_{\mathcal{L}_{0}^{2, \lambda}}\left(Q_{T}\right),
$$

where $\alpha=\frac{\lambda-7}{2}$.

Now we consider the following linear periodical problem:

$$
\begin{aligned}
& \frac{\partial u}{\partial t}-\gamma D^{6} u=\Phi(x, t), \quad(x, t) \in Q_{T}=(0,1) \times(0, T), \\
& \left.u\right|_{x=0,1}=\left.D^{2} u\right|_{x=0,1}=\left.D^{4} u\right|_{x=0,1}=0, \quad t \in(0, T), \\
& u(x, 0)=u(x, T), \quad x \in(0,1) .
\end{aligned}
$$

Here we simply assume that $\Phi(x, t)$ is sufficiently smooth. Our main purpose is to find the relation between the Hölder norm of the solution $u$ and $\Phi(x, t)$.

Let $y_{0}=\left(x_{0}, t_{0}\right) \in \bar{Q}_{T}$ be a fixed point and define

$$
\varphi(u, \rho)=\iint_{S_{\rho}}\left(\left|u(x, t)-\hat{u}_{\rho}\right|^{2}+\rho^{6}\left|D^{3} u(x, t)\right|^{2}\right) d x d t \quad(\rho>0) .
$$

Let $u$ be an arbitrary solution of problem (2.1)-(2.3). We split $u$ on $S_{R}=S_{R}\left(y_{0}\right)$ as $u=u_{1}+u_{2}$ so that $u_{1}$ solves the problem

$$
\begin{aligned}
& \frac{\partial u_{1}}{\partial t}-\gamma D^{6} u_{1}=0, \quad(x, t) \in S_{R}, \\
& \int_{E_{R}} u_{1}(x, t) d x=\int_{E_{R}} u(x, t) d x, \quad t \in(0, T), \\
& \left.u_{1}\right|_{\partial_{1} J_{R}}-\left.u_{1}\right|_{\partial_{2} J_{R}}=\left.u\right|_{\partial_{1} J_{R}}-\left.u\right|_{\partial J_{R}},\left.\quad P^{i}(x, D) u_{1}\right|_{\partial E_{R}}=\left.P^{i}(x, D) u\right|_{\partial E_{R}},
\end{aligned}
$$

and $u_{2}$ solves the problem

$$
\begin{aligned}
& \frac{\partial u_{2}}{\partial t}-\gamma D^{6} u_{2}=\Phi(x, t), \quad(x, t) \in S_{R}, \\
& \int_{E_{R}} u_{2}(x, t) d x=0, \quad t \in(0, T), \\
& \left.u_{2}\right|_{\partial_{1} J_{R}}-\left.u_{2}\right|_{\partial_{2} J_{R}}=\left.P^{i}(x, D) u_{2}\right|_{\partial E_{R}}=0,
\end{aligned}
$$

where

$$
P^{i}(x, D)=\left\{\begin{array}{ll}
D^{i} & \text { if } x=0,1, \\
D^{i+1} & \text { if } x \neq 0,1,
\end{array} \quad i=0,2,4\right.
$$

and $\partial_{1} J_{R}, \partial_{2} J_{R}$ are the down-side and up-side points of $J_{R}$, and $\partial E_{R}$ is the boundary of $E_{R}$.

Some essential estimates on $u_{1}$ and $u_{2}$ are based on the following lemmas.

Lemma 2.2 For the solution $u_{2}$ of problem (2.7)-(2.9), we have

$$
\iint_{S_{R}}\left(D^{i} u_{2}\right)^{2} d x d t+R^{6} \iint_{S_{R}}\left(D^{i+3} u_{2}\right)^{2} d x d t \leq C R^{12-2 i} \iint_{S_{R}} \Phi^{2} d x d t,
$$

where $C$ is a positive constant, $i=0,1,2$. 
Proof Noticing the condition (2.8) and the boundary value condition (2.9), we use the Poincaré inequality and interpolation method (see Chapter 5 in [12]) and get

$$
\begin{aligned}
& \iint_{S_{R}} u_{2}^{2} d x d t \leq C R^{2} \iint_{S_{R}}\left(D u_{2}\right)^{2} d x d t \\
& \iint_{S_{R}}\left(D u_{2}\right)^{2} d x d t \leq \frac{\varepsilon}{R^{2}} \iint_{S_{R}} u_{2}^{2} d x d t+C R^{4} \iint_{S_{R}}\left(D^{3} u_{2}\right)^{2} d x d t
\end{aligned}
$$

which implies that

$$
\iint_{S_{R}} u_{2}^{2} d x d t \leq C R^{6} \iint_{S_{R}}\left(D^{3} u_{2}\right)^{2} d x d t
$$

Multiplying equation (2.7) by $u_{2}$, integrating the result over $S_{R}$ and using the boundary value condition (2.9), we have

$$
\gamma \iint_{S_{R}}\left(D^{3} u_{2}(x, t)\right)^{2} d x d t=\iint_{S_{R}} \Phi(x, t) u_{2}(x, t) d x d t
$$

Using the Young inequality and (2.12), we obtain

$$
\begin{aligned}
\left|\iint_{S_{R}} \Phi(x, t) u_{2}(x, t) d x d t\right| & \leq \frac{\varepsilon}{R^{6}} \iint_{S_{R}} u_{2}^{2} d x d t+C R^{6} \iint_{S_{R}} \Phi^{2} d x d t \\
& \leq \varepsilon \iint_{S_{R}}\left(D^{3} u_{2}\right)^{2} d x d t+C R^{6} \iint_{S_{R}} \Phi^{2} d x d t
\end{aligned}
$$

Combining (2.12), (2.13) and (2.14) yields the estimate (2.10) with $i=0$.

Similarly, multiplying (2.7) by $D^{2} u_{2}$ and $D^{4} u_{2}$, we can obtain the estimates (2.10) with $i=1, i=2$.

Lemma 2.3 For any $\left(x_{1}, t\right),\left(x_{2}, t\right),\left(x, t_{1}\right),\left(x, t_{2}\right) \in S_{\rho}$,

$$
\begin{aligned}
& \left|u_{1}\left(x_{1}, t\right)-u_{1}\left(x_{2}, t\right)\right|^{2} \leq C M\left(u_{1}, \rho\right)\left|x_{1}-x_{2}\right|, \\
& \left|u_{1}\left(x, t_{1}\right)-u_{1}\left(x, t_{2}\right)\right|^{2} \leq C M\left(u_{1}, \rho\right)\left|t_{1}-t_{2}\right|^{1 / 6},
\end{aligned}
$$

where

$$
M\left(u_{1}, \rho\right)=\sup _{t \in J_{\rho}} \int_{E_{\rho}}\left(D u_{1}(x, t)\right)^{2} d x+\iint_{S_{\rho}}\left(D^{4} u_{1}\right)^{2} d x d t,
$$

and $C$ is a constant number. Further, (2.15) and (2.16) still hold if $u_{1}$ is replaced by $D u_{1}$ or $D^{2} u_{1}$.

Proof The estimate (2.15) is obvious. In fact, by the Hölder inequality,

$$
\begin{aligned}
\left|u_{1}\left(x_{1}, t\right)-u_{1}\left(x_{2}, t\right)\right|^{2} & =\left|\int_{x_{1}}^{x_{2}} D u_{1}(x, t) d x\right|^{2} \\
& \leq\left(\int_{x_{1}}^{x_{2}}\left|D u_{1}(x, t)\right| d x\right)^{2} \leq M\left(u_{1}, \rho\right)\left|x_{1}-x_{2}\right| .
\end{aligned}
$$


For (2.16), we only consider the case when $\Delta t=t_{1}-t_{2}>0, x, x+3(\Delta t)^{1 / 6} \in E_{\rho}$. Integrating equation (2.4) over the region $\left(t_{1}, t_{2}\right) \times\left(z, z+(\Delta t)^{1 / 6}\right)$, we have

$$
\int_{z}^{z+(\Delta t)^{1 / 6}}\left[u_{1}\left(\xi, t_{2}\right)-u_{1}\left(\xi, t_{1}\right)\right] d \xi-\gamma \int_{t_{1}}^{t_{2}}\left[D^{5} u_{1}\left(z+(\Delta t)^{1 / 6}, s\right)-D^{5} u_{1}(z, s)\right] d s=0 .
$$

Integrating the above equation with respect to $z$ over $\left(y, y+(\Delta t)^{1 / 6}\right)$, and then integrating the result with respect to $y$ over $\left(x, x+(\Delta t)^{1 / 6}\right)$, we have

$$
\begin{aligned}
& \int_{x}^{x+(\Delta t)^{1 / 6}} \int_{y}^{y+(\Delta t)^{1 / 6}} \int_{z}^{z+(\Delta t)^{1 / 6}}\left[u_{1}\left(\xi, t_{2}\right)-u_{1}\left(\xi, t_{1}\right)\right] d \xi d z d y \\
& =\gamma \int_{t_{1}}^{t_{2}} \int_{x}^{x+(\Delta t)^{1 / 6}}\left[\left(D^{4} u_{1}\left(y+2(\Delta t)^{1 / 6}, s\right)-D^{4} u_{1}\left(y+(\Delta t)^{1 / 6}, s\right)\right)\right. \\
& \left.\quad-\left(D^{4} u_{1}\left(y+(\Delta t)^{1 / 6}, s\right)-D^{4} u_{1}(y, s)\right)\right] d y d s .
\end{aligned}
$$

By virtue of the mean value theorem and the Hölder inequality, we see that there exists $\xi^{*} \in\left(x, x+3(\Delta t)^{1 / 6}\right)$ such that

$$
\begin{aligned}
(\Delta t)^{1 / 2}\left|u_{1}\left(\xi^{*}, t_{1}\right)-u_{1}\left(\xi^{*}, t_{2}\right)\right| & \leq C(\Delta t)^{7 / 12}\left(\iint_{S_{\rho}}\left(D^{4} u_{1}\right)^{2} d x d t\right)^{1 / 2} \\
& \leq C(\Delta t)^{7 / 12}\left(M\left(u_{1}, \rho\right)\right)^{1 / 2}
\end{aligned}
$$

Combining the above result with (2.15), it follows that

$$
\left|u_{1}\left(x, t_{1}\right)-u_{1}\left(x, t_{2}\right)\right|^{2} \leq C M\left(u_{1}, \rho\right)\left|t_{1}-t_{2}\right|^{1 / 6} .
$$

To prove the results on $D u_{1}$ or $D^{2} u_{1}$, we only need to differentiate equation (2.4) once or twice with respect to $x$. And the next procedures are completely similar to the above argument.

\section{Lemma 2.4}

$$
\sup _{t \in J_{\frac{R}{4}}} \int_{E_{\frac{R}{4}}}\left(D^{i} u_{1}\right)^{2} d x+\iint_{S_{\frac{R}{4}}}\left(D^{i+3} u_{1}\right)^{2} d x d t \leq \frac{C}{R^{8}} \iint_{S_{R}}\left(D^{i-1} u_{1}-\lambda\right)^{2} d x d t,
$$

where $C$ is a constant and $i=1,2,3$,

$$
\lambda= \begin{cases}\text { arbitrary constant } & \text { if } Q_{R} \cap \partial_{p} Q_{T}=\emptyset \\ 0 & \text { if } Q_{R} \cap \partial_{p} Q_{T} \neq \emptyset\end{cases}
$$

Proof In order to prove (2.17) with $i=1$, we first prove that

$$
\sup _{t \in J_{\frac{R}{4}}} \int_{E_{\frac{R}{4}}}\left(u_{1}(x, t)-\lambda\right)^{2} d x+\iint_{S_{\frac{R}{4}}}\left(D^{3} u_{1}\right)^{2} d x d t \leq \frac{C}{R^{6}} \iint_{S_{\frac{R}{2}}}\left(u_{1}-\lambda\right)^{2} d x d t .
$$

We discuss it in the following two cases. 
(I) We first prove (2.18) in the case $0, T \in J_{R}$. In such a case, $J_{R}=(0, T), \lambda=0$. Choose a smooth function $\chi(x)$ satisfying the following requirements.

If $0,1 \notin E_{R}$, then supp $\chi \subset\left(x_{0}-\frac{R}{2}, x_{0}+\frac{R}{2}\right), \chi(x)=1$ when $x \in\left(x_{0}-\frac{R}{4}, x_{0}+\frac{R}{4}\right), 0 \leq \chi(x) \leq 1$,

$$
\left|\chi^{\prime}(x)\right| \leq \frac{C}{R}, \quad\left|\chi^{\prime \prime}(x)\right| \leq \frac{C}{R^{2}}, \quad\left|\chi^{\prime \prime \prime}(x)\right| \leq \frac{C}{R^{3}}, \quad\left|\chi^{(4)}(x)\right| \leq \frac{C}{R^{4}}
$$

If $0 \in E_{R}$, then the value of $\chi(x)$ for $x \leq x_{0}$ is changed into 1 .

If $1 \in E_{R}$, then the value of $\chi(x)$ for $x \geq x_{0}$ is changed into 1 .

Multiplying equation (2.4) by $\chi^{6} u_{1}$ and integrating the result over $S_{R}$, then using the boundary value condition (2.6), we have

$$
\begin{aligned}
0= & \iint_{S_{R}} D^{3} u_{1} D^{3}\left(\chi^{6} u_{1}\right) d x d t \\
= & \iint_{S_{R}} \chi^{6}\left(D^{3} u_{1}\right)^{2} d x d t+18 \iint_{S_{R}} \chi^{5} \chi^{\prime} D^{2} u_{1} D^{3} u_{1} d x d t+90 \iint_{S_{R}} \chi^{4} \chi^{\prime 2} D u_{1} D^{3} u_{1} d x d t \\
& +18 \iint_{S_{R}} \chi^{5} \chi^{\prime \prime} D u_{1} D^{3} u_{1} d x d t+\iint_{S_{R}}\left(\chi^{6}\right)^{\prime \prime \prime} u_{1} D^{3} u_{1} d x d t .
\end{aligned}
$$

By the Young inequality and the definition of $\chi(x)$, we have

$$
\begin{aligned}
& 18 \iint_{S_{R}} \chi^{5} \chi^{\prime} D^{2} u_{1} D^{3} u_{1} d x d t \\
& \quad \leq \varepsilon \iint_{S_{R}} \chi^{6}\left(D^{3} u_{1}\right)^{2} d x d t+C \iint_{S_{R}} \chi^{4} \chi^{\prime 2}\left(D^{2} u_{1}\right)^{2} d x d t .
\end{aligned}
$$

Similarly, we can estimate other three terms. Combining the above expressions yields

$$
\begin{aligned}
& \iint_{S_{R}} \chi^{6}\left(D^{3} u_{1}\right)^{2} d x d t \\
& \leq C\left(\iint_{S_{R}} \chi^{2} \chi^{\prime 4}\left(D u_{1}\right)^{2} d x d t+\iint_{S_{R}} \chi^{4} \chi^{\prime \prime 2}\left(D u_{1}\right)^{2} d x d t\right. \\
& \left.\quad+\iint_{S_{R}} \chi^{4} \chi^{\prime 2}\left(D^{2} u_{1}\right)^{2} d x d t+\frac{1}{R^{6}} \iint_{S_{\frac{R}{2}}} u_{1}^{2} d x d t\right) \\
& \equiv C\left(I_{1}+I_{2}+I_{3}+I_{4}\right) .
\end{aligned}
$$

As for $I_{1}$, we have

$$
\begin{aligned}
I_{1} & =-\iint_{S_{R}} u_{1} D\left(\chi^{2} \chi^{\prime 4} D u_{1}\right) d x d t \\
& =-\iint_{S_{R}} \chi^{2} \chi^{\prime 4} u_{1} D^{2} u_{1} d x d t-\iint_{S_{R}}\left(\chi^{2} \chi^{\prime 4}\right)^{\prime} u_{1} D u_{1} d x d t \\
& \leq \varepsilon I_{3}+C \iint_{S_{R}} \chi^{\prime 6} u_{1}^{2} d x d t+\frac{1}{2} \iint_{S_{R}}\left(\chi^{2} \chi^{\prime 4}\right)^{\prime \prime} u_{1}^{2} d x d t \\
& \leq \varepsilon I_{3}+C I_{4} .
\end{aligned}
$$


As for $I_{2}$, we have

$$
\begin{aligned}
I_{2}= & -\iint_{S_{R}} u_{1} D\left(\chi^{4} \chi^{\prime \prime 2} D u_{1}\right) d x d t \\
= & \iint_{S_{R}} \chi^{\prime} D\left(\chi^{4} \chi^{\prime \prime} u_{1} D^{2} u_{1}\right) d x d t+\frac{1}{2} \iint_{S_{R}}\left(\chi^{4} \chi^{\prime \prime 2}\right)^{\prime \prime} u_{1}^{2} d x d t \\
\leq & \iint_{S_{R}} \chi^{\prime}\left(\chi^{4} \chi^{\prime \prime}\right)^{\prime} u_{1} D^{2} u_{1} d x d t \\
& +\iint_{S_{R}} \chi^{4} \chi^{\prime} \chi^{\prime \prime}\left(D u_{1} D^{2} u_{1}+u_{1} D^{3} u_{1}\right) d x d t+C I_{4} \\
= & \varepsilon I_{3}+C I_{4}+\varepsilon \iint_{S_{R}} \chi^{6}\left(D^{3} u_{1}\right)^{2} d x d t-\frac{1}{2} \iint_{S_{R}}\left(\chi^{4} \chi^{\prime} \chi^{\prime \prime}\right)^{\prime}\left(D u_{1}\right)^{2} d x d t \\
= & \varepsilon I_{3}+C I_{4}+\varepsilon \iint_{S_{R}} \chi^{6}\left(D^{3} u_{1}\right)^{2} d x d t-\frac{1}{2} I_{2} \\
& -\frac{1}{2} \iint_{S_{R}}\left(\chi^{4} \chi^{\prime} \chi^{\prime \prime \prime}+4 \chi^{3} \chi^{\prime 2} \chi^{\prime \prime}\right)\left(D u_{1}\right)^{2} d x d t,
\end{aligned}
$$

that is,

$$
\begin{aligned}
I_{2} \leq & \varepsilon I_{3}+C I_{4}+\varepsilon \iint_{S_{R}} \chi^{6}\left(D^{3} u_{1}\right)^{2} d x d t \\
& -\frac{1}{3} \iint_{S_{R}}\left(\chi^{4} \chi^{\prime} \chi^{\prime \prime \prime}+4 \chi^{3} \chi^{\prime 2} \chi^{\prime \prime}\right)\left(D u_{1}\right)^{2} d x d t .
\end{aligned}
$$

On the other hand,

$$
\begin{aligned}
& -\iint_{S_{R}}\left(\chi^{4} \chi^{\prime} \chi^{\prime \prime \prime}+4 \chi^{3} \chi^{\prime 2} \chi^{\prime \prime}\right)\left(D u_{1}\right)^{2} d x d t \\
& =\iint_{S_{R}}\left(\chi^{4} \chi^{\prime} \chi^{\prime \prime \prime}+4 \chi^{3} \chi^{\prime 2} \chi^{\prime \prime}\right) u_{1} D^{2} u_{1} d x d t \\
& \quad+\iint_{S_{R}}\left(\left(\chi^{4} \chi^{\prime} \chi^{\prime \prime \prime}\right)^{\prime}+4\left(\chi^{3} \chi^{\prime 2} \chi^{\prime \prime}\right)^{\prime}\right) u_{1} D u_{1} d x d t \\
& \leq \varepsilon I_{3}+C I_{4} .
\end{aligned}
$$

Combining the above two yields

$$
I_{2} \leq \varepsilon I_{3}+C I_{4}+\varepsilon \iint_{S_{R}} \chi^{6}\left(D^{3} u_{1}\right)^{2} d x d t
$$

Notice that

$$
\begin{aligned}
I_{3}= & -\iint_{S_{R}} \chi^{4} \chi^{\prime 2} D u_{1} D^{3} u_{1} d x d t \\
& -\iint_{S_{R}}\left(4 \chi^{3} \chi^{\prime 3}+2 \chi^{4} \chi^{\prime} \chi^{\prime \prime}\right) D u_{1} D^{2} u_{1} d x d t \\
\leq & \varepsilon \iint_{S_{R}} \chi^{6}\left(D^{3} u_{1}\right)^{2} d x d t+C I_{1}+\varepsilon I_{3}+C I_{1}+\varepsilon I_{3}+C I_{2}
\end{aligned}
$$


that is,

$$
I_{3} \leq C\left(I_{1}+I_{2}\right)+\varepsilon \iint_{S_{R}} \chi^{6}\left(D^{3} u_{1}\right)^{2} d x d t
$$

Finally, from (2.20), (2.21) and (2.22), we see that

$$
I_{i} \leq \varepsilon \iint_{S_{R}} \chi^{6}\left(D^{3} u_{1}\right)^{2} d x d t+C I_{4}, \quad i=1,2,3
$$

which combined with (2.19) yields

$$
\iint_{S_{\frac{R}{4}}}\left(D^{3} u_{1}\right)^{2} d x d t \leq \iint_{S_{R}} \chi^{6}\left(D^{3} u_{1}\right)^{2} d x d t \leq C I_{4}=\frac{C}{R^{6}} \iint_{S_{\frac{R}{2}}} u_{1}^{2} d x d t .
$$

We can imitate all the above procedures and derive a similar result on $E_{R}$, that is,

$$
\left|\int_{E_{R}} D^{3} u_{1} D^{3}\left(\chi^{6} u_{1}\right) d x\right| \leq \int_{E_{R}} H\left(\chi, \chi^{\prime}, \chi^{\prime \prime}, \chi^{\prime \prime \prime}, \chi^{(4)}\right) u_{1}^{2} d x
$$

where $H$ is a polynomial with respect to $\chi, \chi^{\prime}, \chi^{\prime \prime}, \chi^{\prime \prime \prime}, \chi^{(4)}$ and satisfies $|H| \leq \frac{C}{R^{6}}$. Using the Sobolev inequality on $J_{R}$, we have

$$
\begin{aligned}
\sup _{t \in J_{R}} \int_{E_{R}} \chi^{6} u_{1}^{2}(x, t) d x & \leq \frac{C}{R^{6}} \iint_{S_{\frac{R}{2}}} u_{1}^{2} d x d t+2 \int_{J_{R}}\left|\int_{E_{R}} \chi^{6} u_{1} \frac{\partial u_{1}}{\partial t} d x\right| d t \\
& =C I_{4}+2 \gamma \int_{J_{R}}\left|\int_{E_{R}} \chi^{6} u_{1} D^{6} u_{1} d x\right| d t \\
& \leq C I_{4}+C \int_{J_{R}}\left|\int_{E_{R}} D^{3} u_{1} D^{3}\left(\chi^{6} u_{1}\right) d x\right| d t .
\end{aligned}
$$

Combining the above with (2.24) yields

$$
\sup _{t \in J_{\frac{R}{4}}} \int_{E_{\frac{R}{4}}} u_{1}^{2}(x, t) d x \leq \frac{C}{R^{6}} \iint_{S_{\frac{R}{2}}} u_{1}^{2} d x d t .
$$

Combining the above with (2.23) yields the desired estimate (2.18).

(II) Then we prove (2.18) in the case 0 or $T \notin J_{R}$. Take the case $0, T \notin J_{R}$ as an example. Choose another smooth function $\eta(t)$ such that $\eta(t)=1$ when $x \in\left(t_{0}-\left(\frac{R}{4}\right)^{6}, t_{0}+\left(\frac{R}{4}\right)^{6}\right)$; $\eta(t)=0$ when $x \in\left(0, t_{0}-\left(\frac{R}{2}\right)^{6}\right) \cup\left(t_{0}+\left(\frac{R}{2}\right)^{6}, T\right) ; 0 \leq \eta(t) \leq 1 ;\left|\eta^{\prime}(t)\right| \leq \frac{C}{R^{6}}$ for all $t \in(0, T)$.

With $\lambda$ stated in the lemma, we multiply (2.4) by $\chi^{6} \eta\left(u_{1}-\lambda\right)$ and integrate the result over $S_{R}$. Then we can derive equalities similar to the above argument in which $u_{1}$ is replaced by $u_{1}-\lambda$ and a term

$$
-\frac{1}{2} \iint_{S_{R}} \chi^{6} \eta^{\prime}\left(u_{1}-\lambda\right)^{2} d x d t
$$

is added. Then following the argument as in Case I, we can complete the proof of (2.18). 
Now we multiply (2.4) by $D\left(\chi^{6} D u_{1}\right)$ and follow the above argument. Then we derive the same result on $D u_{1}$ :

$$
\sup _{t \in J_{\frac{R}{4}}} \int_{E_{\frac{R}{4}}}\left(D u_{1}(x, t)\right)^{2} d x+\iint_{S_{\frac{R}{4}}}\left(D^{4} u_{1}\right)^{2} d x d t \leq \frac{C}{R^{6}} \iint_{S_{\frac{R}{2}}}\left(D u_{1}\right)^{2} d x d t .
$$

Using the interpolation inequality, we have

$$
\iint_{S_{\frac{R}{2}}}\left(D u_{1}\right)^{2} d x d t \leq \frac{C}{R^{2}} \iint_{S_{\frac{R}{2}}} u_{1}^{2} d x d t+C R^{4} \iint_{S_{\frac{R}{2}}}\left(D^{3} u_{1}\right)^{2} d x d t .
$$

Replacing $R$ in (2.23) by $2 R$, and combining the result with the above inequality, we have

$$
\iint_{S_{\frac{R}{2}}}\left(D u_{1}\right)^{2} d x d t \leq \frac{C}{R^{2}} \iint_{S_{R}} u_{1}^{2} d x d t
$$

which together with (2.25) yields (2.17) with $i=1$.

For (2.17) with $i=2$ and $i=3$, we should first multiply (2.4) by $D\left(\chi^{6} D u_{1}\right)$ and $D^{2}\left(\chi^{6} D^{2} u_{1}\right)$ respectively, and the remaining parts are similar and easier.

Lemma 2.5 For any $0<\rho<R$,

$$
\varphi\left(u_{1}, \rho\right) \leq C\left(\frac{\rho}{R}\right)^{8} \varphi\left(u_{1}, R\right)
$$

where $C$ is a constant number. Further, (2.26) still holds, if $u_{1}$ is replaced by $D u_{1}$ or $D^{2} u_{1}$.

Proof It suffices to show (2.26) for $\rho \leq \frac{R}{4}$, otherwise we only need to set $C=4^{8}$. By Lemma 2.3 and Lemma 2.4, we have

$$
\iint_{S_{\rho}}\left|u_{1}-\hat{u}_{1_{\rho}}\right|^{2} d x d t \leq C M\left(u_{1}, \frac{R}{4}\right) \rho^{8} \leq C\left(\frac{\rho}{R}\right)^{8} \iint_{S_{R}}\left(u_{1}-\lambda\right)^{2} d x d t .
$$

Taking $\lambda=\hat{u}_{1_{R}}$, we obtain

$$
\iint_{S_{\rho}}\left|u_{1}-\hat{u}_{1_{\rho}}\right|^{2} d x d t \leq C\left(\frac{\rho}{R}\right)^{8} \iint_{S_{R}}\left(u_{1}-\hat{u}_{1_{R}}\right)^{2} d x d t .
$$

On the other hand, by (2.25),

$$
\begin{aligned}
& \iint_{S_{\rho}} \rho^{6}\left(D^{3} u_{1}(x, t)\right)^{2} d x d t \\
& \quad \leq C \iint_{S_{\rho}} \rho^{2}\left(D u_{1}(x, t)\right)^{2} d x d t+C \iint_{S_{\rho}} \rho^{8}\left(D^{4} u_{1}(x, t)\right)^{2} d x d t \\
& \quad \leq C\left(\frac{\rho}{R}\right)^{8}\left[\iint_{S_{R}}\left|u_{1}-\hat{u}_{1_{\rho}}\right|^{2} d x d t+\iint_{S_{R}} R^{6}\left(D^{3} u_{1}\right)^{2} d x d t\right] \\
& \quad=C\left(\frac{\rho}{R}\right)^{8} \varphi\left(u_{1}, R\right),
\end{aligned}
$$


which combined with (2.27) implies (2.26). The proofs of the results on $D u_{1}$ or $D^{2} u_{1}$ are similar.

Lemma 2.6 Let $\varphi(\rho)$ be a nonnegative and nondecreasing function satisfying

$$
\varphi(\rho) \leq A\left(\frac{\rho}{R}\right)^{\alpha} \varphi(R)+B R^{\beta}, \quad 0<\rho \leq R \leq R_{0}
$$

where $A, B, \alpha, \beta$ are positive constants and $\beta<\alpha$. Then there exists a constant $C$ only depending on $A, B, \alpha, \beta$ such that

$$
\varphi(\rho) \leq C\left(\frac{\rho}{R}\right)^{\beta}\left[\varphi(R)+B R^{\beta}\right], \quad 0<\rho \leq R \leq R_{0} .
$$

The proof of this lemma can be found in [13].

Theorem 2.1 Let $\Phi(x, t)$ be an appropriately smooth function, and let $u$ be the smooth solution of problem (2.1)-(2.3). Then, for any $\alpha \in\left(0, \frac{1}{2}\right)$, there exists a coefficient $K$ depending only on $\alpha, \iint_{Q_{T}} u^{2} d x d t, \iint_{Q_{T}}\left(D^{3} u\right)^{2} d x d t, \iint_{Q_{T}} \Phi^{2} d x d t$ such that

$$
\left|u\left(x_{1}, t_{1}\right)-u\left(x_{2}, t_{2}\right)\right| \leq K\left(\left|x_{1}-x_{2}\right|^{\alpha}+\left|t_{1}-t_{2}\right|^{\frac{\alpha}{\sigma}}\right) .
$$

Further, (2.28) still holds if $u$ is replaced by $D u$ or $D^{2} u$.

Proof For any fixed point $\left(x_{0}, t_{0}\right) \in \bar{Q}_{T}$, consider the function $\varphi(u, \rho)$, which is clearly nondecreasing with respect to $\rho$. By Lemma 2.5,

$$
\begin{aligned}
\varphi(u, \rho) & \leq \varphi\left(u_{1}, \rho\right)+\varphi\left(u_{2}, \rho\right) \\
& \leq C\left(\frac{\rho}{R}\right)^{8} \varphi\left(u_{1}, R\right)+\varphi\left(u_{2}, R\right) \\
& \leq C\left(\frac{\rho}{R}\right)^{8} \varphi(u, R)+C \varphi\left(u_{2}, R\right)
\end{aligned}
$$

holds for any $0<\rho<R$. By Lemma 2.2,

$$
\begin{aligned}
\varphi\left(u_{2}, R\right) & =\iint_{S_{R}}\left[\left(u_{2}-\hat{u}_{2_{R}}\right)^{2}+R^{6}\left(D^{3} u_{2}\right)^{2}\right] d x d t \\
& \leq 4 \iint_{S_{R}} u_{2}^{2} d x d t+R^{6} \iint_{S_{R}}\left(D^{3} u_{2}\right)^{2} d x d t \\
& \leq C R^{12} \iint_{S_{R}} \Phi^{2} d x d t .
\end{aligned}
$$

Thus,

$$
\varphi(u, \rho) \leq C\left(\frac{\rho}{R}\right)^{8} \varphi(u, R)+C R^{12} \iint_{Q_{T}} \Phi^{2} d x d t .
$$


By Lemma 2.6, we have

$$
\varphi(u, \rho) \leq C\left(\frac{\rho}{R_{0}}\right)^{\lambda}\left[\varphi\left(u, R_{0}\right)+R_{0}^{\lambda} \iint_{Q_{T}} \Phi^{2} d x d t\right]
$$

for some $7<\lambda<8$. Hence,

$$
M^{2}[u] \leq C\left[\frac{1}{R_{0}^{\lambda}} \varphi\left(u, R_{0}\right)+\iint_{Q_{T}} \Phi^{2} d x d t\right] .
$$

Using Lemma 2.1, we immediately obtain (2.28). The proofs of the results on $D u_{1}$ or $D^{2} u_{1}$ are similar.

\section{The main result and its proof}

In this section, we represent the main result of this paper.

Theorem 3.1 Problem (1.1)-(1.3) admits a time-periodic solution $u \in C^{6+\alpha, 1+\frac{\alpha}{6}}(\bar{Q})$.

To prove the existence of this solution, we employ the Leray-Schauder fixed point theorem which enables us to study the problem by considering the following equation:

$$
\frac{\partial u}{\partial t}-\gamma D^{6} u=\sigma D g(x, t)+\sigma f(x, t)
$$

subject to the conditions (1.2)-(1.3), where $\sigma$ is a parameter taking value on the interval $[0,1]$, and $g(x, t) \in \mathcal{W}$ is periodic in time $t$ with period $T$, where $\mathcal{W} \equiv\{w \mid w \in$ $C^{1+\alpha, \frac{\alpha}{4}}\left(\bar{Q}_{T}\right), w(x, t)$ is periodic in time $t$ with period $\left.T\right\}$. For any given function $g(x, t) \in \mathcal{W}$, from linear classical theory (see [14]), we see that problems (3.1) and (1.2)-(1.3) admit a unique solution $u \in C^{6+\alpha, 1+\frac{\alpha}{6}}\left(\bar{Q}_{T}\right) \subset C^{1+\alpha, \frac{\alpha}{4}}\left(\bar{Q}_{T}\right)$, and hence we can define a mapping $\mathcal{L}$ as follows:

$$
\mathcal{L}: \mathcal{W} \times[0,1] \rightarrow \mathcal{W}, \quad(g, \sigma) \mapsto u,
$$

together with its composition with $\Psi(v, t)=D^{3} \psi(v, t)+v v^{2}$, namely

$$
\mathcal{L}(\Psi(\cdot, \cdot), \cdot): \mathcal{W} \times[0,1] \rightarrow \mathcal{W}
$$

Obviously, for any given $v \in \mathcal{W}, \mathcal{L}(v, 0)=0$. By virtue of the Leray-Schauder fixed point theorem, to prove the existence of solutions of problem (1.1)-(1.3), we only need to show that the mapping $\mathcal{L}$ is compact and prove that there exists a constant independent of $u_{\sigma}$ and $\sigma$ such that, for any $u$ and $\sigma$ satisfying $u=\mathcal{L}(\Psi(u), \sigma),\left\|u_{\sigma}\right\|_{C^{4+\alpha, \frac{\alpha}{4}}\left(\bar{Q}_{T}\right)} \leq C$. Moreover, it follows from the above arguments that $u$ is a classical solution. Then we consider the problem in $Q_{(T, 2 T)}, \ldots, Q_{((n-1) T, n T)}, \ldots$ in turn. Finally, we know that initial boundary value problem (1.1)-(1.3) admits a classical solution in $Q$.

Lemma 3.1 The mapping $\mathcal{L}:(v, \sigma) \mapsto u$ is compact.

This result can be directly obtained by a compact embedding theorem, so we omit the details here. 
Lemma 3.2 Let $u_{\sigma}$ be a time-periodic solution of the equation

$$
\frac{\partial u_{\sigma}}{\partial t}-\gamma D^{6} u_{\sigma}=\sigma D^{4} \psi\left(u_{\sigma}, t\right)+\sigma v u_{\sigma} D u_{\sigma}+\sigma f(x, t),
$$

subject to the conditions (1.2)-(1.3), where $\sigma \in[0,1]$. Then

$$
\left\|u_{\sigma}\right\|_{\infty} \leq C, \quad\left\|D u_{\sigma}\right\|_{\infty} \leq C, \quad\left\|D^{2} u_{\sigma}\right\|_{\infty} \leq C,
$$

where $C$ is a constant independent of the solution $u$ and $\sigma$.

Proof First, let $\omega_{\sigma}(x, t)$ be a time-periodic solution of the problem

$$
D^{2} \omega=u_{\sigma},\left.\quad D \omega\right|_{x=0,1}=0, \quad \int_{0}^{1} \omega d x=0,
$$

then from the Poincaré inequality we know that

$$
\int_{0}^{1} \omega_{\sigma}^{2} d x \leq \int_{0}^{1}\left(D \omega_{\sigma}\right)^{2} d x \leq \int_{0}^{1}\left(D^{2} \omega_{\sigma}\right)^{2} d x=\int_{0}^{1} u_{\sigma}^{2} d x .
$$

Multiplying (3.2) by $\omega_{\sigma}(x, t)$, integrating the result over $Q_{T}$ and using the condition (1.2), then using the Young inequality and (3.4), we have

$$
\begin{aligned}
& \gamma \iint_{Q_{T}}\left(D^{2} u_{\sigma}\right)^{2} d x d t+\sigma \iint_{Q_{T}} 3 a(t) u_{\sigma}^{2}\left(D u_{\sigma}\right)^{2} d x d t \\
& \quad=\sigma \iint_{Q_{T}} b(t)\left(D u_{\sigma}\right)^{2} d x d t-\sigma \iint_{Q_{T}} v u_{\sigma} D u_{\sigma} \omega_{\sigma} d x d t-\sigma \iint_{Q_{T}} f(x, t) \omega_{\sigma} d x d t \\
& \quad \leq C \iint_{Q_{T}}\left(D u_{\sigma}\right)^{2} d x d t+\frac{M}{2} \sigma \int_{Q_{T}} u_{\sigma}^{2}\left(D u_{\sigma}\right)^{2} d x d t+C \iint_{Q_{T}} \omega_{\sigma}^{2} d x d t+C,
\end{aligned}
$$

which implies that

$$
\iint_{Q_{T}}\left(D^{2} u_{\sigma}\right)^{2} d x d t \leq C \iint_{Q_{T}} u_{\sigma}^{2} d x d t+C .
$$

Moreover,

$$
\begin{aligned}
\iint_{Q_{T}}\left(D u_{\sigma}\right)^{2} d x d t & =-\iint_{Q_{T}} u_{\sigma} D^{2} u_{\sigma} d x d t \\
& \leq C \iint_{Q_{T}}\left(D^{2} u_{\sigma}\right)^{2} d x d t+C \iint_{Q_{T}} u_{\sigma}^{2} d x d t \\
& \leq C \iint_{Q_{T}} u_{\sigma}^{2} d x d t+C .
\end{aligned}
$$

It follows from (3.5) that

$$
\begin{aligned}
\iint_{Q_{T}} u_{\sigma}^{2}\left(D u_{\sigma}\right)^{2} d x d t & \leq \frac{N}{3 \underline{M}} \iint_{Q_{T}}\left(D u_{\sigma}\right)^{2} d x d t+\frac{\Lambda}{3 \underline{M}} \iint_{Q_{T}} \omega_{\sigma}^{2} d x d t+C \\
& \leq C \iint_{Q_{T}} u_{\sigma}^{2} d x d t+C .
\end{aligned}
$$


By (1.2), we have

$$
u_{\sigma}^{4}(x, t)=\int_{0}^{x} D\left(u_{\sigma}^{4}(s, t)\right) d s \leq \int_{0}^{1}\left|D u_{\sigma}^{4}\right| d x=4 \int_{0}^{1}\left|u_{\sigma}^{3} D u_{\sigma}\right| d x
$$

Integrating the above inequality over $Q_{T}$ and using (3.8) together with the Young inequality, we have

$$
\begin{aligned}
\iint_{Q_{T}} u_{\sigma}^{4} d x d t & \leq 4 \iint_{Q_{T}}\left|u_{\sigma}^{3} D u_{\sigma}\right| d x d t \\
& \leq 4\left(\iint_{Q_{T}} u_{\sigma}^{4} d x d t\right)^{1 / 2}\left(\iint_{Q_{T}} u_{\sigma}^{2}\left(D u_{\sigma}\right)^{2} d x d t\right)^{1 / 2} \\
& \leq 4\left(\iint_{Q_{T}} u_{\sigma}^{4} d x d t\right)^{1 / 2}\left(C \iint_{Q_{T}} u_{\sigma}^{2} d x d t+C\right)^{1 / 2}
\end{aligned}
$$

that is,

$$
\iint_{Q_{T}} u_{\sigma}^{4} d x d t \leq C \iint_{Q_{T}} u_{\sigma}^{2} d x d t+C
$$

On the other hand, by the Young inequality,

$$
\iint_{Q_{T}} u_{\sigma}^{2} d x d t \leq \varepsilon \iint_{Q_{T}} u_{\sigma}^{4} d x d t+C
$$

Combining the above expressions, we obtain

$$
\iint_{Q_{T}} u_{\sigma}^{4} d x d t \leq C, \quad \iint_{Q_{T}} u_{\sigma}^{2} d x d t \leq C .
$$

Combining the above with (3.6) and (3.7), we see that

$$
\iint_{Q_{T}}\left(D u_{\sigma}\right)^{2} d x d t \leq C, \quad \iint_{Q_{T}}\left(D^{2} u_{\sigma}\right)^{2} d x d t \leq C .
$$

Set

$$
F(t)=\int_{0}^{1}\left[\frac{\gamma}{2}\left(D u_{\sigma}\right)^{2}+\sigma\left(H\left(u_{\sigma}, t\right)+\lambda\right)\right] d x
$$

where $H(u, t)=-\int_{0}^{u} \psi(s, t) d s=\frac{a(t)}{4} u^{4}-\frac{b(t)}{2} u^{2} \geq-\lambda, \lambda$ is a positive constant depending only on $\underline{M}$ and $N$. Then $F(t) \geq 0$. Integrating $|F(t)|$ over $(0, T)$, by (3.9) and (3.10), we get

$$
\begin{aligned}
\int_{0}^{T}|F(t)| d t & \leq \iint_{Q_{T}}\left|\frac{\gamma}{2}\left(D u_{\sigma}\right)^{2}+\sigma\left(\frac{a(t)}{4} u^{4}-\frac{b(t)}{2} u^{2}+\lambda\right)\right| d x d t \\
& \leq C \iint_{Q_{T}}\left(D u_{\sigma}\right)^{2} d x d t+C \iint_{Q_{T}} u_{\sigma}^{4} d x d t+C \iint_{Q_{T}} u_{\sigma}^{2} d x d t+C \\
& \leq C .
\end{aligned}
$$


On the other hand, integrating by parts and using (1.2), we have

$$
\begin{aligned}
\frac{d F}{d t}= & -\int_{0}^{1}\left(\gamma D^{4} u_{\sigma}+\sigma D^{2} \psi\right)^{2} d x-\sigma \int_{0}^{1}\left(\gamma D^{2} u_{\sigma}+\sigma \psi\right) v u_{\sigma} D u_{\sigma} d x \\
& -\sigma \int_{0}^{1}\left(\gamma D^{2} u_{\sigma}+\sigma \psi\right) f d x+\sigma \int_{0}^{1}\left(\frac{a^{\prime}(t)}{4} u_{\sigma}^{4}-\frac{b^{\prime}(t)}{2} u_{\sigma}^{2}\right) d x
\end{aligned}
$$

Integrating the above equality over $(0, T)$ and noticing the periodicity of $F$, we have

$$
\begin{aligned}
& \iint_{Q_{T}}\left(\gamma D^{4} u_{\sigma}+\sigma D^{2} \psi\right)^{2} d x d t \\
& =-\sigma \iint_{Q_{T}}\left(\gamma D^{2} u_{\sigma}+\sigma \psi\right) v u_{\sigma} D u_{\sigma} d x d t-\sigma \iint_{Q_{T}}\left(\gamma D^{2} u_{\sigma}+\sigma \psi\right) f d x d t \\
& \quad+\sigma \iint_{Q_{T}}\left(\frac{a^{\prime}(t)}{4} u_{\sigma}^{4}-\frac{b^{\prime}(t)}{2} u_{\sigma}^{2}\right) d x d t
\end{aligned}
$$

Integrating $\left|\frac{d F}{d t}\right|$ over $(0, T)$, using (3.9) and (3.10), we have

$$
\begin{aligned}
& \int_{0}^{T}\left|\frac{d F}{d t}\right| d t \\
& \leq 2 \iint_{Q_{T}}\left|\left(\gamma D^{2} u_{\sigma}+\sigma \psi\right) \nu u_{\sigma} D u_{\sigma}\right| d x d t+2 \iint_{Q_{T}}\left|\left(\gamma D^{2} u_{\sigma}+\sigma \psi\right) f\right| d x d t \\
& \quad+2 \iint_{Q_{T}}\left|\frac{a^{\prime}(t)}{4} u_{\sigma}^{4}-\frac{b^{\prime}(t)}{2} u_{\sigma}^{2}\right| d x d t \\
& \leq C \iint_{Q_{T}}\left(D^{2} u_{\sigma}\right)^{2} d x d t+C \iint_{Q_{T}} u_{\sigma}^{4} d x d t+C \iint_{Q_{T}}\left|u_{\sigma}\right|^{3} d x d t \\
& \quad+C \iint_{Q_{T}} u_{\sigma}^{2} d x d t+C \leq C .
\end{aligned}
$$

By virtue of (3.11) and (3.12), we have $F(t) \leq C$. Noticing the definition of $F(t)$, we get

$$
\int_{0}^{1}\left(D u_{\sigma}\right)^{2} d x \leq C
$$

By (1.2), we know that $\left\|u_{\sigma}\right\|_{\infty} \leq C$.

In order to prove the rest of this lemma, we need to give a priori estimate on $D^{4} u_{\sigma}^{3}$. First, by the Gagliardo-Nirenberg inequality, we can obtain

$$
\begin{gathered}
\left\|D u_{\sigma}\right\|_{4} \leq C\left\|D^{6} u_{\sigma}\right\|_{2}^{\frac{1}{20}}\left\|D u_{\sigma}\right\|_{2}^{\frac{19}{20}}, \\
\left\|D u_{\sigma}\right\|_{8} \leq C\left\|D^{6} u_{\sigma}\right\|_{2}^{\frac{3}{40}}\left\|D u_{\sigma}\right\|_{2}^{\frac{37}{40}}, \\
\left\|D^{2} u_{\sigma}\right\|_{4} \leq C\left\|D^{6} u_{\sigma}\right\|_{2}^{\frac{1}{4}}\left\|D u_{\sigma}\right\|_{2}^{\frac{3}{4}}, \\
\left\|D^{3} u_{\sigma}\right\|_{4} \leq C\left\|D^{6} u_{\sigma}\right\|_{2}^{\frac{9}{20}}\left\|D u_{\sigma}\right\|_{2}^{\frac{11}{20}},
\end{gathered}
$$


Lin et al. Boundary Value Problems 2013, 2013:73

Page 15 of 17

where $\|*\|_{p}$ denotes the $L_{p}$ norm on $(0,1)$. Regulating the exponents and using the Young inequality for every of the above three expressions, we get

$$
\begin{aligned}
& \int_{0}^{1}\left(D u_{\sigma}\right)^{4} d x \leq \varepsilon \int_{0}^{1}\left(D^{6} u_{\sigma}\right)^{2} d x+C\left(\int_{0}^{1}\left(D u_{\sigma}\right)^{2} d x\right)^{19 / 9} \\
& \int_{0}^{1}\left(D u_{\sigma}\right)^{8} d x \leq \varepsilon \int_{0}^{1}\left(D^{6} u_{\sigma}\right)^{2} d x+C\left(\int_{0}^{1}\left(D u_{\sigma}\right)^{2} d x\right)^{37 / 7} \\
& \int_{0}^{1}\left(D^{2} u_{\sigma}\right)^{4} d x \leq \varepsilon \int_{0}^{1}\left(D^{6} u_{\sigma}\right)^{2} d x+C\left(\int_{0}^{1}\left(D u_{\sigma}\right)^{2} d x\right)^{3} \\
& \int_{0}^{1}\left(D^{3} u_{\sigma}\right)^{4} d x \leq \varepsilon \int_{0}^{1}\left(D^{6} u_{\sigma}\right)^{2} d x+C\left(\int_{0}^{1}\left(D u_{\sigma}\right)^{2} d x\right)^{11}
\end{aligned}
$$

Integrating the above inequalities over $(0, T)$ and noticing (3.13), we see that the terms of left hand side in these inequalities can all be estimated by $\varepsilon \iint_{Q_{T}}\left(D^{6} u_{\sigma}\right)^{2} d x d t$ and a constant number $C$. Then by the boundary value condition and (3.10), we have

$$
\iint_{Q_{T}}\left(D^{4} u_{\sigma}\right)^{2} d x d t \leq \varepsilon \iint_{Q_{T}}\left(D^{6} u_{\sigma}\right)^{2} d x d t+C,
$$

and also, by the above discussion, we have

$$
\begin{aligned}
\iint_{Q_{T}}\left(D^{4} u_{\sigma}^{3}\right)^{2} d x d t \leq & C \iint_{Q_{T}}\left(D^{4} u_{\sigma}\right)^{2} d x d t+C \iint_{Q_{T}}\left(D u_{\sigma} D^{3} u_{\sigma}\right)^{2} d x d t \\
& +C \iint_{Q_{T}}\left(\left|D u_{\sigma}\right|^{2} D^{2} u_{\sigma}\right)^{2} d x d t+C \iint_{Q_{T}}\left(\left|D^{2} u_{\sigma}\right|^{2}\right)^{2} d x d t \\
\leq & \varepsilon \iint_{Q_{T}}\left(D^{6} u_{\sigma}\right)^{2} d x d t+C
\end{aligned}
$$

Multiplying (3.2) by $D^{6} u_{\sigma}$, integrating the result over $Q_{T}$, using (3.14), (3.15) and the Young inequality, we get

$$
\begin{aligned}
\iint_{Q_{T}}\left(D^{6} u_{\sigma}\right)^{2} d x d t & \leq \varepsilon \iint_{Q_{T}}\left(D^{6} u_{\sigma}\right)^{2} d x d t+C \iint_{Q_{T}}\left(D^{4} \psi\left(u_{\sigma}, t\right)\right)^{2} d x d t+C \\
& \leq \varepsilon \iint_{Q_{T}}\left(D^{6} u_{\sigma}\right)^{2} d x d t+C,
\end{aligned}
$$

that is,

$$
\iint_{Q_{T}}\left(D^{6} u_{\sigma}\right)^{2} d x d t \leq C
$$

By (3.17) and the approach similar to (3.14), we can derive

$$
\begin{aligned}
& \iint_{Q_{T}}\left(D^{3} u_{\sigma}\right)^{2} d x d t \leq C, \quad \iint_{Q_{T}}\left(D^{4} u_{\sigma}\right)^{2} d x d t \leq C, \\
& \iint_{Q_{T}}\left(D^{5} u_{\sigma}\right)^{2} d x d t \leq C .
\end{aligned}
$$


Now we set

$$
F_{1}(t)=\int_{0}^{1}\left(D^{2} u_{\sigma}\right)^{2} d x
$$

Obviously,

$$
\int_{0}^{T}\left|F_{1}(t)\right| d t \leq C .
$$

On the other hand, by (3.16), (3.17) and (3.18), we have

$$
\begin{aligned}
\int_{0}^{T}\left|\frac{d F_{1}}{d t}\right| d t= & \int_{0}^{T}\left|\int_{0}^{1} D^{4} u_{\sigma}\left(\gamma D^{6} u_{\sigma}+\sigma D^{4} \psi+\sigma v u_{\sigma} D u_{\sigma}+\sigma f\right) d x\right| d t \\
\leq & C \iint_{Q_{T}}\left(D^{4} u_{\sigma}\right)^{2} d x d t+C \iint_{Q_{T}}\left(D^{6} u_{\sigma}\right)^{2} d x d t \\
& +C \iint_{Q_{T}}\left(D^{4} \psi\right)^{2} d x d t+C \leq C .
\end{aligned}
$$

By virtue of (3.19) and (3.20), we have $F_{1}(t) \leq C$. Noticing the definition of $F_{1}(t)$, we get

$$
\int_{0}^{1}\left(D^{2} u_{\sigma}\right)^{2} d x \leq C .
$$

Applying the Poincaré inequality and the Friedrichs inequality [15], we conclude that $\left\|D u_{\sigma}\right\|_{\infty} \leq C$.

Finally, we set

$$
F_{2}(t)=\int_{0}^{1}\left(D^{3} u_{\sigma}\right)^{2} d x
$$

By an approach similar to the above argument, we can obtain the last result that $\left\|D^{2} u_{\sigma}\right\|_{\infty} \leq C$. The proof of this lemma is complete.

Proof of Theorem 3.1 Now we apply Theorem 2.1 to complete the proof of Theorem 3.1. For the smooth function $\Phi(x, t)$ in Theorem 2.1 , let

$$
\Phi(x, t)=\sigma D^{4} \psi\left(u_{\sigma}, t\right)+\sigma v u_{\sigma} D u_{\sigma}+\sigma f
$$

From the proof of Lemma 3.2, we see that $\iint_{Q_{T}}\left(D^{i} u_{\sigma}\right)^{2} d x d t(i=0,1, \ldots, 6)$ and $\iint_{Q_{T}} \Phi^{2} d x d t$ can be all uniformly bounded by a constant number $C$. Therefore the coefficient $K$ in Theorem 2.1 now only depends on the Hölder exponent $\alpha$. So, for $u_{\sigma}$, we have

$$
\left|D^{i} u_{\sigma}\left(x_{1}, t_{1}\right)-D^{i} u_{\sigma}\left(x_{2}, t_{2}\right)\right| \leq K(\alpha)\left(\left|x_{1}-x_{2}\right|^{\alpha}+\left|t_{1}-t_{2}\right|^{\frac{\alpha}{6}}\right), \quad i=0,1,2
$$

which combines with the results of Lemma 3.2. We know that $\left\|u_{\sigma}\right\|_{C^{2+\alpha}, \frac{\alpha}{6}\left(\bar{Q}_{T}\right)} \leq C$, where $C$ is independent of $u$ and $\sigma$. Then, it follows from the results in [16] that $\left\|u_{\sigma}\right\|_{C^{4+\alpha, \frac{\alpha}{6}\left(\bar{Q}_{T}\right)}} \leq C$. 
Recalling the discourse in the beginning of this section, we conclude from the LeraySchauder fixed point theorem that $\mathcal{L}(*, 1)$ admits a fixed point $u$ in the space $C^{6+\alpha, 1+\frac{\alpha}{6}}\left(\bar{Q}_{T}\right)$, which is the desired solution of problem (1.1)-(1.3). The proof of Theorem 3.1 is completed.

\section{Competing interests}

The authors declare that they have no competing interests.

\section{Authors' contributions}

All authors contributed equally to the manuscript and read and approved the final manuscript.

\section{Acknowledgements}

The authors would like to express their deep thanks to the referees for their valuable suggestions, for the revision and improvement of the manuscript. This research was partly supported by the Scientific Research Foundation for the Returned Overseas Chinese Scholars, State Education Ministry.

Received: 6 November 2012 Accepted: 14 March 2013 Published: 4 April 2013

\section{References}

1. Savina, TV, Golovin, AA, Davis, SH: Faceting of a growing crystal surface by surface diffusion. Phys. Rev. E 67, 021606 (2003)

2. Cohen, DS, Murray, JD: A generalized diffusion model for growth and dispersal in a population. J. Math. Biol. 12, 237-249 (1981)

3. Korzec, MD, Evans, PL, Münch, A, Wagner, B: Stationary solutions of driven fourth-and sixth-order Cahn-Hilliard-type equations. SIAM J. Appl. Math. 69, 348-374 (2008)

4. Liu, C, Tang, H, Liu, A: The existence of global attractor for a sixth order parabolic equation. Politehn. Univ. Bucharest Sci. Bull. Ser. A, Appl. Math. Phys. (in press)

5. Evans, JD, Galaktionov, VA, King, JR: Unstable sixth-order thin film equation: I. Blow-up similarity solutions Nonlinearity 20,1799-1841 (2007)

6. Evans, JD, Galaktionov, VA, King, JR: Unstable sixth-order thin film equation: II. Global similarity patterns. Nonlinearity 20, 1843-1881 (2007)

7. Liu, C: Qualitative properties for a sixth-order thin film equation. Math. Model. Anal. 15, 457-471 (2010)

8. Wang, YP, Zhang, YL: Time-periodic solutions to a nonlinear parabolic type equation of higher order. Acta Math. Appl. Sin. 24, 129-140 (2008)

9. Yin, L, Li, Y, Huang, R, Yin, J: Time periodic solutions for a Cahn-Hilliard type equation. Math. Comput. Model. 48, 11-18 (2008)

10. Gilbarg, D, Trudinger, N: Elliptic Partial Differential Equations of Second Order, 2nd edn. Springer, Berlin (1983)

11. Yin, J: On the Cahn-Hilliard equation with nonlinear principal part. J. Partial Differ. Equ. 7, 77-96 (1994)

12. Adams, RA, Fournier, JJF: Sobolev Spaces, 2nd edn. Pure and Applied Mathematics, vol. 140. Academic Press, Amsterdam (2003)

13. Giaquinta, M: Multiple Integrals in the Calculus of Variations and Nonlinear Elliptic System. Princeton University Press, Princeton (1983)

14. Ladyzhenskaja, OA, Solonikov, VA, Ural'ceva, NN: Linear and Quasilinear Equations of Parabolic Type. Am. Math. Soc. Providence (1968)

15. Elliott, CM, Zheng, SM: On the Cahn-Hilliard equation. Arch. Ration. Mech. Anal. 96, 339-357 (1986)

16. Wang, R: The Schauder theory of the boundary value problem for parabolic problem equations. Acta Sci. Nat. Univ. Jilin 2, 35-64 (1964)

doi:10.1186/1687-2770-2013-73

Cite this article as: Liu et al.: Time-periodic solutions for a driven sixth-order Cahn-Hilliard type equation. Boundary Value Problems 2013 2013:73. 